\title{
A TRIAL SPECTACLE FOR PRISM PRESCRIPTION
}

BY

\section{C. Michaelson \\ GLASGOW}

THE purpose of this variable prism spectacle is to determine the prism necessary for the comfort of the patient and to do so by overcoming two difficulties inherent in prism prescription.

The first is the difficulty of knowing to what extent a vertical or horizontal phoria should be neutralised. Some oculists prescribe the full vertical error divided between both eyes, while others prescribe empirically only a fraction of the error, likewise with exophoria for reading distance. Some oculists have no definite ruling by which they prescribe, while others prescribe up to two-thirds of the negative duction at reading distance. The correction for muscular imbalance, in its very nature, cannot have the precise predetermination of the correction for astigmatism.

The second difficulty is that with muscle-imbalance we are not dealing with a static defect measurable with reasonable error at any time, but with a changing defect, the amount of which varies not only with the initial defect and duction power but with the behaviour of these during the constant use of the eyes as in reading. No a priori consideration can inform us of the rate of

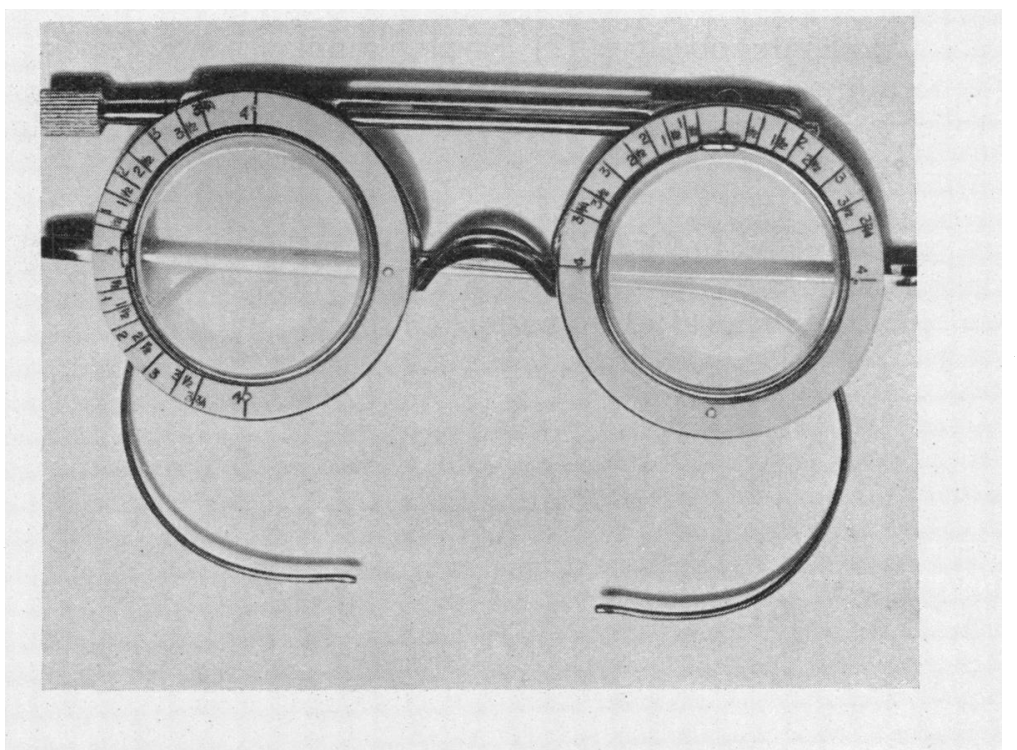


tiredness of the duction powers of the eyes. Because of the failure to recognise these inherent difficulties the prescription of prisms has in many cases, especially with horizontal prisms, been one of trial or error; with the results that comfort is more often given to the patient by the removal rather than by the prescription of the prism and the oculist becomes more hardened in his opinion that prisms do no good.

This spectacle obviates the necessity of determining beforehand the amount of the phoria to be neutralised, and is a means of measuring the changing prismatic needs, which are dependent on the variations in phoria and muscle duction power during the constant and natural use of the eyes.

Each cell of the frame contains a $4^{\Delta}$ prism and both prisms can be simultaneously rotated by the screw so as to give a maximum of $8^{\Delta}$ between both eyes, base in, or base out. A prism can easily be removed and replaced so that a vertical prismatic power up to $8 \Delta$ can be used. The frame is so light that it can be borne without any discomfort over the patient's usual spectacles containing his presbyopic or other correction. The suspicion that a prism may be required is usually aroused when the patient complains that no relief of his symptoms followed the use of his glasses. This, and the type of prism likely to be needed, is confirmed by testing the phorias and duction powers for both distance and near. The patient is given the spectacle to take home and is informed beforehand in which direction to turn the screw and is told to do so when the symptom arises for which prismatic correction is considered to be possibly advisable. This is usually asthenopia and it is not unusual to find a patient who despite the most careful correction of his refraction error cannot read for more than half an hour at a time. He is told to turn the screw about 1 dioptre or so at a time and to continue reading for some time after each change of the prism, until he has reached a point where the maximum comfort seems to have been obtained. This is repeated for several evenings and the result reported at the end of that time to the oculist. The latter has then definite evidence on which to prescribe prisms and knows not to do so should the patient inform him that no help was obtained in any position.

I have not used the instrument yet in treating symptoms due to vertical phorias but many exophorias with low negative duction at reading distance have been treated successfully, and with the confidence its simplicity permits.

The instrument can be used for measuring most of the vertical phorias likely to be met with in practice, and low degrees of horizontal phoria. (The spectacle is supplied by Messrs. Curry and Paxton, L.td.) 